\title{
22. Lunar Rocks as Meteoroid Detectors
}

\author{
JACK B. Hartung and Friedrich Hörz \\ Manned Spacecraft Center, NASA \\ Houston, Texas \\ AND \\ Donald E. GaUlt \\ Ames Research Center, NASA \\ Moffett Field, California
}

\begin{abstract}
About 5000 microcraters on seven lunar rocks recovered during the A pollo 12 mission have been systematically studied using a stereomicroscope. Based on comparisons with laboratory cratering experiments, at least 95 percent of all millimeter-sized craters observed were formed by impacts in which the impact velocity exceeded $10 \mathrm{~km} / \mathrm{s}$. The dynamics of particle motion near the Moon and the distribution of microcraters on the rocks require an extralunar origin for these impacting particles.
\end{abstract}

The microcrater population on at least one side of all rocks studied was in equilibrium for millimeter-sized craters; i.e., statistically, craters a few millimeters in diameter and smaller were being removed by the superposition of new craters at the same rate new craters were being formed. Selected surfaces of some rocks, particularly those with glass coatings, are not in equilibrium. For every particle incident upon these "production" surfaces, there remains for observation a corresponding crater; thus the population of craters on such a surface is directly related to the total population of particles impacting that surface.

Crater size-distribution data from production surfaces, together with an experimentally determined relationship between the crater size and the physical parameters of the impacting particle, yield the mass distribution of the interplanetary dust at $1 \mathrm{AU}$. Based on assumptions corresponding to an impact velocity of about $20 \mathrm{~km} / \mathrm{s}$ and $a$ particle density of $3 \mathrm{~g} / \mathrm{cm}^{3}$, the cumulative particle flux versus mass distribution relationship is

$$
\log \mathrm{N}=-0.5 \log \mathrm{m}+\mathrm{C} \quad \text { for } 10^{-8}<\mathrm{m}<10^{-6} \mathrm{~g}
$$

where $\mathrm{N}$ is the number of particles of mass $\mathrm{m}$ in grams, and larger, and $\mathrm{C}$ depends on the time-area product, which is, for the present, unknown. For particles smaller than $10^{-8} \mathrm{~g}$, our observations indicate a sharper decrease in the absolute value of the slope of the flux versus mass curve than is indicated by satellite-borne-experiment data. This result may be due to a genuine relative decrease in the number or kinetic energy of smaller particles, or it may be due to our inability to observe quantitatively the smallest microcraters. For particles larger than $10^{-6} \mathrm{~g}$, the slope of the flux versus mass curve increases smoothly to an absolute value greater than one. 
$T_{\mathrm{w}}^{\mathrm{H}}$ HE STUDY OF TINY SOLID PARTICLES moving within the solar system-meteoroids-has a long and interesting history. Initially, analysis of visible light streaks in the night sky by astronomers provided the basis for most of what was known about meteoroids. More recently, radio electromagnetic radiation reflected from ionized particles produced during entry of a meteoroid into the atmosphere has been studied using radar techniques. Photometric analysis of the zodiacal light has led to some information about the population of interplanetary particles. With the artificial satellite came a more direct means of detecting meteoroids, and now, very sophisticated electronic devices are used for these studies. The objectives of these efforts have been to determine the mass, velocity, composition, and number density or flux of the particles.

Our purpose in this paper is to discuss a new method that may be applied to the study of meteoroids. That method is the use of exposed lunar rock surfaces as meteoroid detectors. The approach is similar to that of other workers who have studied craters on the actual surface of the Moon that were formed by much larger interplanetary bodies (Shoemaker et al., 1970; Gault, 1970; Hartmann, 1970).

The operation of a lunar rock meteoroid detector is quite simple. A lunar rock surface exposed to space will suffer the impact of interplanetary particles. Each impact produces a small crater on the rock surface. Each erater may be considered a geologic signal, which corresponds to an electronic signal from a satellite detector or to a visible light signal on a photographic plate. Although the operation of a lunar rock as an instrument is simple, as in other experimental methods, its calibration and the analysis and interpretation of data obtained are difficult.

At least one breakthrough has been made in connection with the development of the lunar rock meteoroid detector. In satellite detectors, considerable effort has been expended to maximize the number of events detected. In other words, a large time-area product for the instrument was considered desirable. An estimate of a typical timearea product for a lunar rock meteoroid detector is made in the following manner. The surface area of an exposed face of a lunar rock may be taken to be $100 \mathrm{~cm}^{2}$ or $10^{-2} \mathrm{~m}^{2}$. Cosmic-ray exposure times for whole lunar rocks from $3 \times 10^{7}$ to $5 \times 10^{8} \mathrm{yr}$ based on measurements of spallation rare gases have been determined by several workers and summarized by Bogard et al. (1971). Exposure times of $10^{5}$ to $5 \times 10^{7} \mathrm{yr}$ have been determined by Crozaz et al. (1970), Fleischer et al. (1970), Lal et al. (1970), and Price and O'Sullivan (1970) based on measurements of energetic nuclear particle track densities. Also, exposure times of $10^{5}$ to $10^{6} \mathrm{yr}$ have been inferred for rock 10017 by Shedlovsky et al. (1970) based on the analysis of the radioactive nuclides $\mathrm{Al}^{26}$ and $\mathrm{Mn}^{53}$, which are produced by the interaction of energetic solar flare particles with certain stable nuclides in lunar rocks. For the purposes of this example, a relatively low exposure time of $10^{6}$ years may be taken. This results in a time-area product for the lunar rock meteoroid detector of about $10^{4} \mathrm{~m}^{2}-\mathrm{yr}$, which is several orders of magnitude greater than the value for artificial satellite-borne instruments.

Unfortunately, this relatively low time-area product for typical lunar rocks is still far greater than the optimum for this experiment. The problem is one of retaining a record of only a portion of all events actually occurring on a rock surface, because after a sufficient exposure time, from a statistical viewpoint, the addition of new craters causes the destruction of an equal number of previously existing craters. Most surfaces have reached this state of maturity with respect to cratering and thus are termed "equilibrium" surfaces. A typical crystalline lunar rock with an equilibrium crater population is shown in figure 1 .

It is possible to avoid this problem by carefully selecting a lunar rock surface which has not reached equilibrium, that is, a "production" surface, or one upon which there exists essentially one crater or signal corresponding to each particle that impacted the surface. Rocks with such surfaces do exist among those so far returned from the Moon, but they are rare and not easily obtained for analysis. An example of a production surface is the glass-coated surface of rock 12054 , a portion of which is shown in figure 2 .

\section{MICROCRATER DESCRIPTION}

Observations of lunar rock meteoroid detector surfaces were made using an optical stereoscopic microscope with an available range of magnifica- 


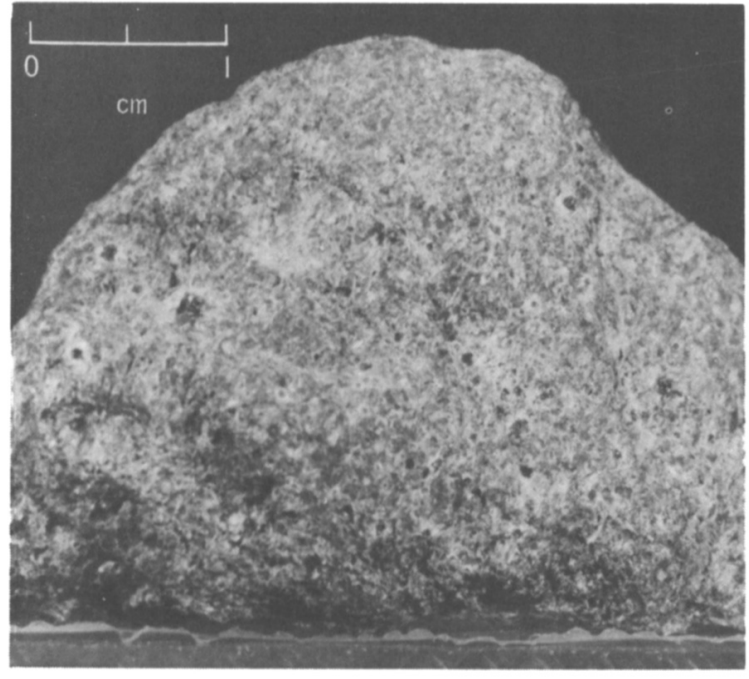

Figure 1.-The surface of rock 12017, shown here, has a typical microcrater density of 10 to 20 microcraters with pits larger than $0.2 \mathrm{~mm} / \mathrm{cm}^{2}$. This surface is in equilibrium with respect to the microcratering process. The largest craters may be recognized by the darkcolored dots (glass-lined pits) surrounded by lightcolored areas (halo material). Most of the craters are too small to be recognized on the scale of this photograph. (NASA MSC photograph 70-45307)

tion of from $3.2 \times$ to $200 \times$. Essentially all observations were made while working at magnifications of between $10 \times$ and $100 \times$. The observational procedure used consisted of first performing a reconnaissance study of the entire rock to gain familiarity with the interesting features and problems related to a particular rock. Then more detailed observations were made. Quantitative data were taken by selecting a field of view of known size which was judged to be representative of the surface and then measuring the important parameters defined by Hörz et al. (1971b) for each crater observed in that field of view. The location of each field of view was indicated on a whole-rock photograph or model. Following this, a completely new field of view was selected and the process repeated until each face of a rock had been thoroughly studied. Faces of rocks were distinguished, based largely on the geometry of the rock.

Microcraters on lunar rocks have been described by LSPET (1969), Neukum et al. (1970), Hörz et al. (1971a, 1971b), and Bloch et al. (1971a). These features may be described in terms of three

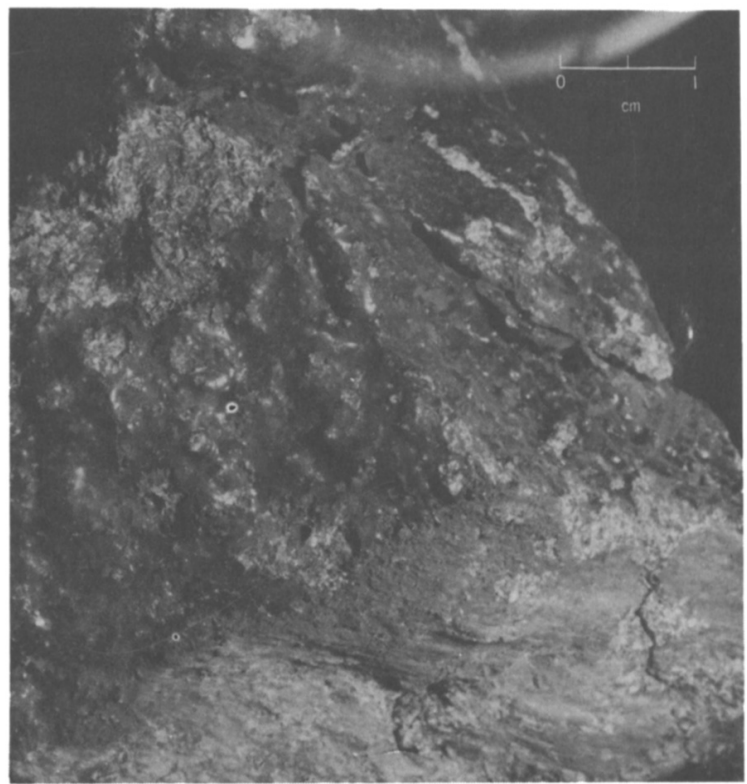

Figure 2.-A portion of the glass-coated surface of rock 12054 is shown here. The glass coating shows the effects of a relatively short period of meteoroid bombardment. The surface has not yet reached equilibrium with respect to microcratering; otherwise, the glass coating would have been removed. The largest cratering events penetrate the glass coating and cause the spalling away of the entire thickness of the coating, thus exposing the light-colored underlying rock. The smaller craters do not penetrate the glass and can be observed as light dots with diameters as small as the resolution limit of the photograph; however, the presence of dust particles on the rock surface makes positive identification difficult at this scale. (NASA MSC photograph 70-22995)

major elements: a central glass-lined pit, a surrounding halo zone consisting of intensely microfractured crystalline material, and a roughly concentric spall zone. The stereoscopic photographs of figure 3 show a portion of an exceptionally large and fresh microcrater which illustrates the typical relationship between these three elements. The diameter of the halo zone is usually from 2 to 2.5 times that of the glass-lined pit. The diameter of the spall area is most often 2.5 to 5 times that of the pit. The ratio of spall diameter to pit diameter decreases as pit size decreases for small craters in glass. For example, on the glass coating of rock 12054 the average spall to pit diameter ratio was about 3 for 200-micron-diameter pits. For 50-micron-diameter pits, this ratio 

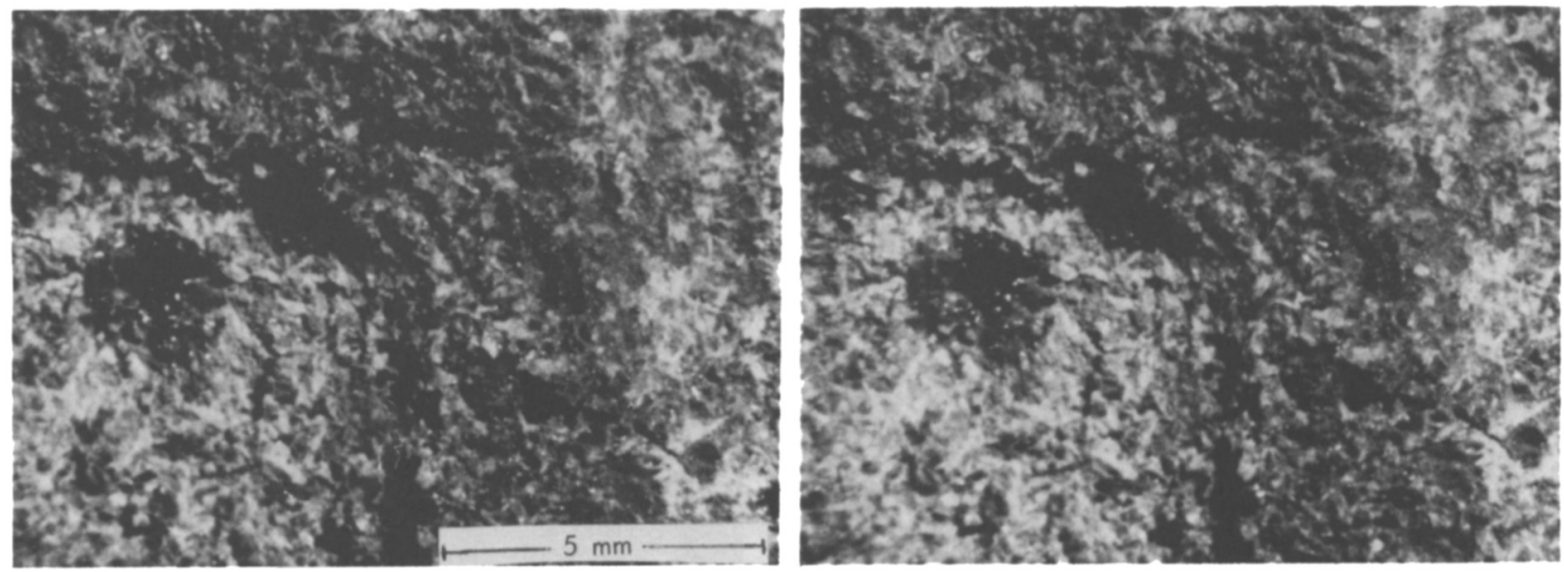

Figure 3.-A portion of an exceptionally large and fresh microcrater is shown in this stereoscopic view. The dark glass-lined pit is surrounded and underlain by lighter crystalline or "halo" material which is thoroughly microfractured. The spall area is delineated by a scarp surrounding the pit and halo. Radially outward, a portion of a concentric ring of darker material is shown, and beyond that is the normal rock surface which displays numerous smaller craters, two of which are easily recognized at the lower right of the photograph. The darker ring is attributed to a very thin layer of condensed silicate vapor produced during the impact event which formed the large microcrater. (NASA MSC photographs 70-29946 and 70-29947).

averaged about 2.5. Pit depths are quite variable but are normally one-fifth to one-half the pit diameter.

A scanning electron micrograph of a single typical small microcrater on a glass fragment is shown in figure 4 . On equilibrium rock surfaces, the spall areas are relatively larger, and those areas for adjacent craters overlap and tend to destroy one another. On such surfaces, only the most recent craters have easily identifiable spall areas.

The glass that lines most pits appears to be derived from the melting of the host rock, based on the usual similarity in the color of the host minerals and the color of the glass linings, especially for the smaller craters. In general, the glass that lines larger pits appears darker. These observations do not rule out the possibility that melted material from the impacting particle has been incorporated in the glass linings.

Recently, Carter and McKay (1971) have produced glass-lined pits by impact at velocities of $7 \mathrm{~km} / \mathrm{s}$ in laboratory experiments by raising the temperature of the target material. Bloch et al. (1971b) and Mandeville and Vedder (1971) have produced similar, but much smaller (micronsized), pits using Van de Graaff microparticle accelerators. For all these experiments, the impact velocity required to produce glass-lined pits is much greater than the $2-\mathrm{km} / \mathrm{s}$ escape velocity for the Moon. Therefore, we have concluded that, in general, such pits were formed by the impact of extralunar or interplanetary micrometeoroids.

Although the great majority, over 95 percent, of the impact features observed on lunar rock surfaces are of the glass-lined-pit type described previously, other types of features do exist and represent sources of possible spurious signals. The production of most glass-lined pits is a process of mass removal from the rock surface. Occasionally, a similar appearing feature is observed which is clearly the result of a mass-addition process. In these cases, a dark glass mass has evidently been deposited on the surface. These features are more irregular in outline and are found in greater numbers near the soil line on a rock. We attribute these features to the secondary "splashing on" of liquid ejecta produced during small impacts in the soil near the rock.

On certain rocks, particularly fine-grained crystalline rocks, a relatively large number of clearly identifiable impact craters are observed which do not possess a central glass-lined pit. These craters are recognized by the existence of a depression lined with thoroughly microfractured halo material and occasionally surrounded by an 


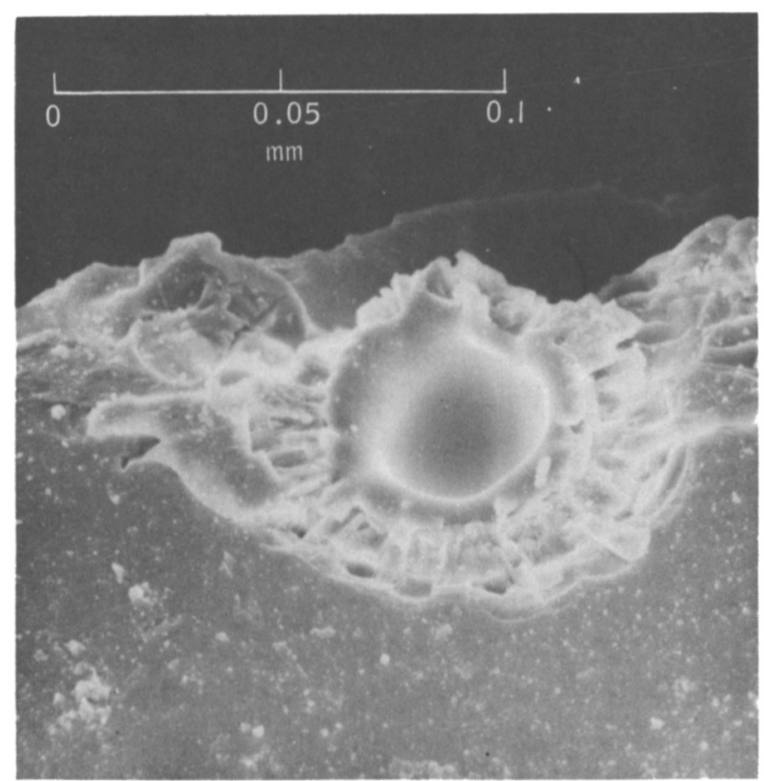

Figure 4.-A scanning electron micrograph of a small microcrater. The glass-lined pit and the spall area are nicely illustrated, but the microfractured halo zone is essentially invisible because the scanning electron microscope technique produces an image of the topography only and does not record any albedo differences present on the surface under study. In this case, the host rock is a millimeter-sized glass fragment from the lunar soil collected during the Apollo 11 mission. (Scanning electron micrograph courtesy of D. S. McKay, NASA MSC photograph 70-40177)

observable spall area. Craters in this group appear on the average larger than the coexisting glasslined-pit-type craters in the same area. We are working on the hypothesis that these pitless craters are produced by the impact of slightly larger meteoroids; and, because of somewhat different mechanical properties of the host rock, the pits are destroyed in the cratering process, while similar impacts on a different rock would not cause destruction of the larger pits. Alternatively, these craters may be the product of impacts by solid particles, either secondary or primary, moving at somewhat lower relative velocities at impact.

In addition to the difficulty in distinguishing between impact craters produced by primary interplanetary particles and secondary particles originating on the Moon, other problems exist that serve to degrade the quality of the statistical data obtained. These problems, taken as a group, compose the overall recognition problem. An example is that the glass lining a small pit occurring in single-mineral grain is often the same color as the host grain, thus making recognition difficult. Small craters in the halo zones of larger craters are not easily observed because the halo of the small crater does not contrast with its surroundings. Microcrater halos are also not well developed on already strongly microfractured whole rocks or on the extremely fine-grained breccias. The highly irregular surface on the microscopic scale of essentially all rocks also contributes to the recognition problem. Finally, lunar rocks are partially coated by "welded dust" (Hörz et al., 1971a), loose dust particles, and other material while resting on the lunar surface and by lunar soil during the collection and processing of the rocks. Rocks are generally cleaned of loosely adhering material, using a gas jet arrangement before our observations are made, but often, dust-filled depressions or a scattering of fine dust remains on the surface after cleaning. Our procedures do not at present include additional cleaning of whole rock surfaces.

Fortunately, the recognition problem may be avoided or at least reduced greatly by the very careful selection of the lunar sample surface to be used as a meteoroid detector. We have found that craters with diameters as low as a few tens of microns are readily recognizable on the glass coatings of certain lunar rocks. Both from the standpoint of obtaining a production population of microcraters and of minimizing the recognition problems, a glass coating on a rock surface is by far the best meteoroid detector.

\section{MICROCRATER POPULATION DATA}

The basic quantitative data obtained in the course of this study to date consist of the areal density and size distribution of microcraters. Because of the way our microcraters, or geologic signals, are analyzed, there is a tendency to underestimate the actual number of events detected. We do not count a crater, or receive a signal, unless it is recognized and identified as corresponding to a definite impact event. In contrast to some meteoroid detection experiments, if an error exists for our experiment, it is on the side of failing to observe an event rather than observing too many events. Consequently, our 
data represent minimum values for the actual number of events recorded.

Shown in figure 5 is a log-log graph of the cumulative areal number density of craters versus crater size as indicated by the diameter of the glass-lined pit for eight different rocks. For each rock, several faces were analyzed, but only the data for the faces yielding the highest crater densities are plotted on this summary plot for the reasons indicated previously. A more detailed presentation of the data for all these rocks except 12054 has been given by Hörz et al. (1971b).

Several points related to the data presented in figure 5 are important. First, the maximum crater densities for all rocks except 12054 are essentially the same, within a factor of 2 , for pit diameters near 0.2 to $0.4 \mathrm{~mm}$. When an appropriate spall area for each crater is considered, this value for crater density is about 10 percent of saturation (as defined by Gault, 1970). This level of saturation is higher than that for most regolith-covered areas on the lunar surface which have been studied on a larger scale and shown to be equi- librium surfaces with respect to cratering (Shoemaker et al., 1970; Gault, 1970).

The probable reason a higher level of saturation exists on rock surfaces than on the regolith is that craters on rock surfaces are removed only by superposition of new craters, while craters on the regolith are, in addition, filled in by the sedimentation of ejecta material from nearby, but not necessarily superimposing, cratering events (Soderblom, 1970). However, in general, these considerations lead to the view that the most densely cratered surfaces of all rocks studied so far, except 12054, are in equilibrium with respect to cratering. The cratering on the thin glass coating of rock 12054 has obviously not yet reached equilibrium; otherwise, the delicate glass coating itself would be completely ruptured and destroyed.

The curves in figure 5 for all rocks except 12054 begin to flatten, that is, the absolute value of the slope decreases, for craters with pit diameters below about $0.2 \mathrm{~mm}$. The curve for rock 12054 remains relatively steep down to a pit diameter of

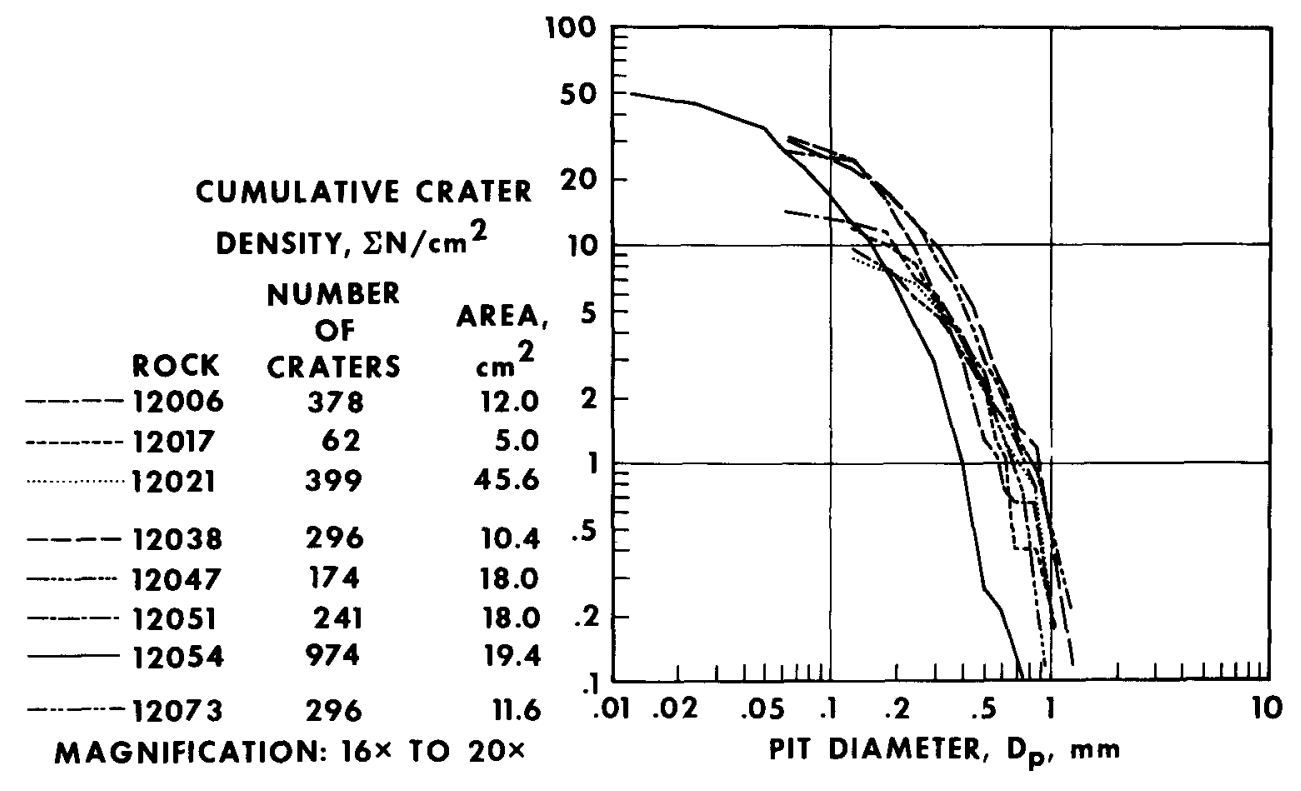

Figure 5.-The cumulative areal crater density versus crater size is shown for several lunar rocks. For each rock, the face having the maximum crater density is shown. Similar maximum crater densities for all rocks except 12054 suggest these rock surfaces have reached equilibrium with respect to the cratering process. The flattening of the curves for all rocks except 12054 at smaller crater sizes illustrates the recognition problem that exists for most rocks. The curve for rock 12054 extends to smaller crater sizes, indicating the superior "sensing" qualities of glass-coated surfaces. 
$0.05 \mathrm{~mm}$. This comparison illustrates the recognition problem relative to the smaller craters on crystalline rock and breccia surfaces. The presence of a relatively large number of $0.05-\mathrm{mm}$ pits on rock 12054 indicates that a similar relative number of smaller events must have occurred on the other rocks, but that the craters formed have simply not been recognized.

The curves in figure 5 appear to steepen as larger crater sizes are approached. Unfortunately, the number of large events observed is insufficient to permit a definite conclusion based on these data. Several possible explanations are considered by Hörz et al. (1971b).

Of particular interest is the flattening of the curve at smaller crater sizes for rock 12054 . This flattening may, with no further consideration, be attributed to the recognition problem, which, for a glass-coated surface, simply shifts the flattening to smaller crater sizes, where the observational problems again become dominant. However, we believe, based only on qualitative data obtained during microscopic study of rock 12054, that the flattening of this curve is, at least in part, attributable to a genuine relative decrease in the number or energy of smaller particles impacting the surface of the rock. It will be shown later that the particle size at which this tendency toward fewer events occurs agrees generally with results obtained independently by other investigators of meteoroids.

The data obtained for rock 12054 are presented in detail in figure 6 . The data corresponding to two independent investigators working at magnifications of $20 \times, 40 \times$, and $100 \times$ are indicated. The procedure for viewing at different magnifications was to select a field of view at $20 \times$, take data at that magnification, increase the magnification to $40 \times$ without moving the sample, take data at $40 \times$, increase the magnification to $100 \times$ also without moving the sample, and take data at $100 \times$. Occasionally, the $100 \times$ field of view would
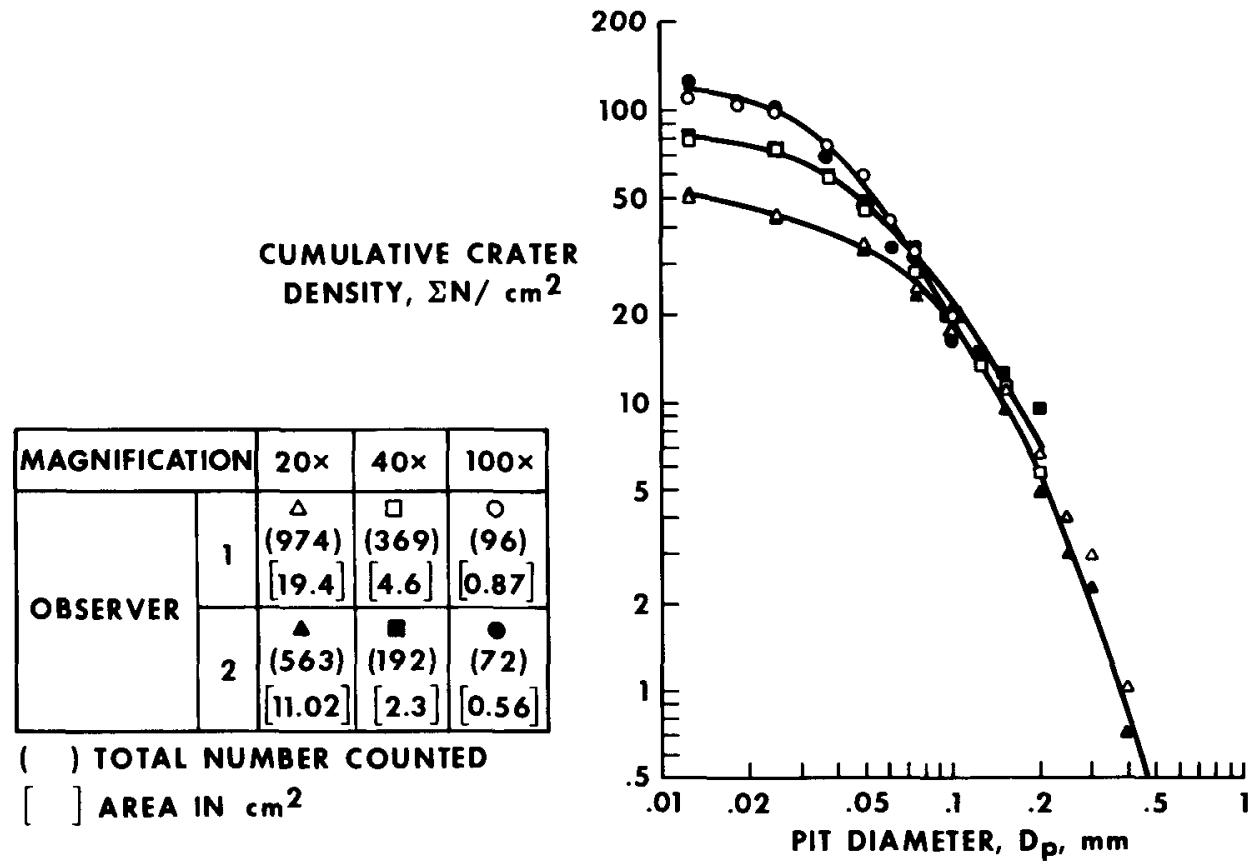

Figure 6.-Microcrater density and size data are shown for rock 12054. Results of independent study by two observers at three different magnifications are indicated. The recognition problem is further illustrated by the successively greater densities of smaller craters observed at successively higher magnifications. However, based on qualitative observations, the flattening of the envelope of the curves shown may be in part due to a genuine relative decrease in the number or energy of the smaller interplanetary particles. The curve shown here for a magnification of $20 \times$ is the same as the one shown for rock 12054 in figure 5 . 
be rejected because it fell in an area of extremely poor viewing conditions or in the spall area of a single larger crater. Therefore, the data for $100 \times$ magnification may be considered to be "selected" to obtain the maximum crater densities present.

Fields of view counted by one observer were not intentionally duplicated by the other observer. Agreement between the two different workers is within the limits of probable error at essentially all magnifications. This shows a lack of bias on the part of the observers and a uniformity of the crater population over different parts of the surface studied.

The recognition problem also exists for glass surfaces, as is well illustrated by the different positions at which flattening of the curves occurs. The flattening begins at larger crater sizes when lower magnification is used The crater diameter at which flattening occurs is well above the resolution limit of the microscope. We conclude that where the curves at successive magnifications agree, the data are accurate and free from the recognition problem. The recognition problems experienced in our microscopic studies are similar to those experienced in the evaluation of lunar surface photography at various levels of resolution (Shoemaker et al., 1970).

However, we argue that the flattening of the curve for a magnification of $100 \times$ is, at least in part, an effect due to a genuine decrease in the relative number or energy of micrometeoroids making the smallest craters (less than 0.05-mm pit diameter), based on the following evidence. When a glass surface is viewed at a magnification of $20 \times$, for example, a number of minute features exist which may or may not be impact craters. A judgment is required to decide whether these features should be considered craters. At a magnification of $100 \times$, when observing conditions are good, the abundance of such features is significantly reduced. The curves, of course, reflect this depletion, but the appearance is the same as would exist if the recognition problem were the entire explanation for the flattening of the curve for a magnification of $100 \times$. The uncertainty described here will be eliminated when observations can be made on an especially prepared surface of rock 12054 , using both an optical and a scanning electron microscope.

\section{DETECTOR CALIBRATION STUDIES}

To relate quantitatively the geologic signals or microcraters described so far to meteoroids or interplanetary dust requires a sizable ground-based calibration effort. In spite of all the hypervelocity impact experiments that have been undertaken, the development of a well-calibrated lunar rock meteoroid detector is just beginning. The objectives of such a calibration are the determination of the mass, velocity, shape, and composition of individual meteoroids.

Laboratory experiments by Vedder (1971), Bloch et al. (1971b), and Mandeville and Vedder (1971) using Van de Graaff microparticle accelerators provide at present the basis for such a calibration. In these experiments, particles with masses in the range $5 \times 10^{-13}$ to $5 \times 10^{-9} \mathrm{~g}$ were accelerated to velocities as high as $30 \mathrm{~km} / \mathrm{s}$, though most of the data were obtained for impact velocities below $10 \mathrm{~km} / \mathrm{s}$. Various glasses, crystalline materials, and rock materials were used as targets. Projectiles were polystyrene, density = $1.08 \mathrm{~g} / \mathrm{cm}^{3}$, and iron, density $=7.87 \mathrm{~g} / \mathrm{cm}^{3}$. These projectile and target materials represent fairly well the boundary conditions anticipated for lunar rock meteoroid detectors on the lunar surface. However, at present, experimental limitations do not permit simulation of the range of particle masses and/or sizes necessary to evaluate quantitatively the lunar microcraters under study. The experimental projectiles, 0.1 to 6 microns in diameter, produced craters only a few microns in diameter, whereas the craters of interest on lunar rocks are tens to hundreds of microns in diameter. We must, therefore, extrapolate the experimental parameters over several orders of magnitude.

The fundamental problem consists of relating measurable parameters associated with the crater to the important characteristics of the impacting projectile, which are as follows:

(1) Size $\}$ (If any two are known, the

(2) Density (3) third may be determined.)

(4) Shape

(5) Velocity (magnitude and direction)

Because all experiments have so far used spherical projectiles, we will assume spherical projectile geometry. Mandeville and Vedder (1971) indicate that for oblique impacts, the crater depth 
and threshold for spallation are determined by the normal component of velocity, while the asymmetry of the crater is controlled by the tangential component. Because the effect of the velocity direction on the size of the glass-lined pit has not yet been clearly determined, we may assume the velocity parameter to be either the normal component or the total impact velocity.

Two approaches to the calibration of the lunar rock meteoroid detector have been suggested. The first relies on the result of both sets of experiments that over the projectile mass range studied for a constant projectile density, the ratio of the glass-lined pit diameter, $D_{p}$, to the projectile diameter, $d$, is very nearly independent of the projectile size or mass, $m$. This ratio, $D_{p} / d$, does, however, vary with the projectile impact velocity (Mandeville and Vedder, 1971; Bloch et al., 1971b). This variation for both groups of experiments is shown in figure 7 . Thus, using these curves for a given impact velocity and a given projectile density, $\rho$ we may estimate a single value of $D_{p} / d$ and calculate the mass, $m$, of a projectile which formed a pit of diameter, $D_{p}$, by use of the equation

$$
m=\frac{\pi \rho}{6}\left(\frac{D_{p}}{D_{p} / d}\right)^{3}
$$

where $m$ is in grams, $\rho$ is in $\mathrm{g} / \mathrm{cm}^{3}$, and $D_{p}$ is in centimeters.

The second possible approach is based on the

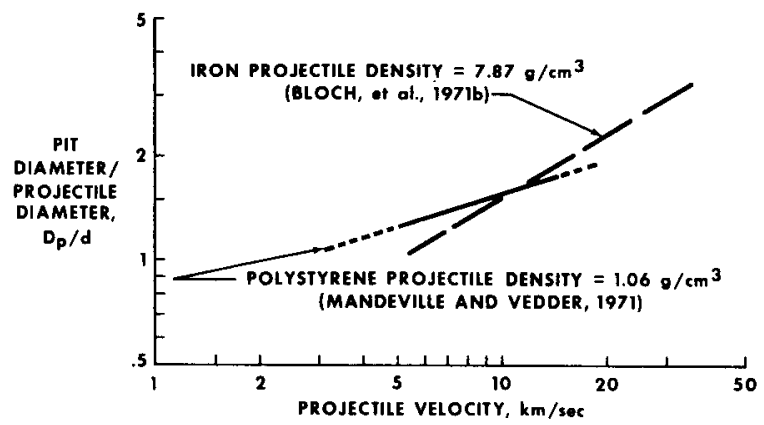

Figure 7.-A summary of available experimental cratering data is shown. In this study, 0.1- to 6-micron iron or polystyrene particles were accelerated, using Van de Graaff microparticle accelerators. Target materials were silicate glass or crystalline material with densities of about $2.5 \mathrm{~g} / \mathrm{cm}^{3}$. relationship that exists between the kinetic energy of the impacting projectile and the mass of the target material displaced during the cratering event. With this approach, the mass of the projectile would be determined by measuring the volume of the crater, finding the required kinetic energy through use of the experimentally derived relationship, and assuming some average impact velocity.

An essential difference between these two approaches is that the first requires a pit diameter measurement and the second requires a pit volume measurement or estimate based on a model crater geometry. The advantage of the first approach is that no pit geometry model is required as long as the pit diameter is a welldefined parameter. The second approach offers the possible advantage of permitting a $D_{p} / d$ which is not necessarily constant for all sizes of particles. Because neither accurate measurements of pit volumes nor careful crater geometry determinations have been made, for the purposes of this paper we have chosen the first approach to solve the calibration problem.

\section{METEOROID POPULATION DATA}

Using equation (1), we have converted the crater size distribution data corresponding to the envelope of the curves in figure 6 to particle mass distribution curves which are shown in figure $8(a)$. Because the $D_{p} / d$ ratio varies with projectile velocity and density, as indicated in figure 7 , curves are presented for several different values of that ratio. This method of presentation also allows visualization of mass distribution curves that would represent the situation where $D_{p} / d$ did in fact increase with increasing projectile size, other parameters being held constant. An increase of $D_{p} / d$ with increasing projectile kinetic energy is suggested from crater scaling laws (Moore et al., 1964). However, at present, no quantitative data on the actual magnitude of $D_{p} / d$ variations are available.

Unfortunately, the data presented in figure 8 (a) do not yield an independent determination of the absolute flux of meteoroids impacting the lunar rock meteoroid detector because, as yet, no appropriate exposure time data have been obtained for this rock surface. However, we can 


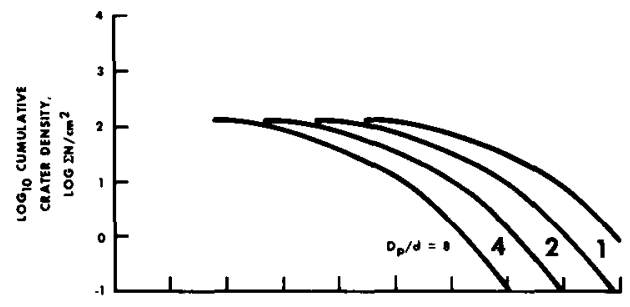

(a) ROCK 12054 DATA

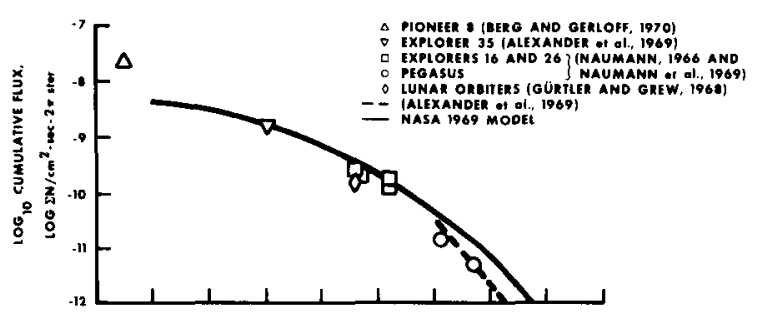

(b) SATELLITE DATA

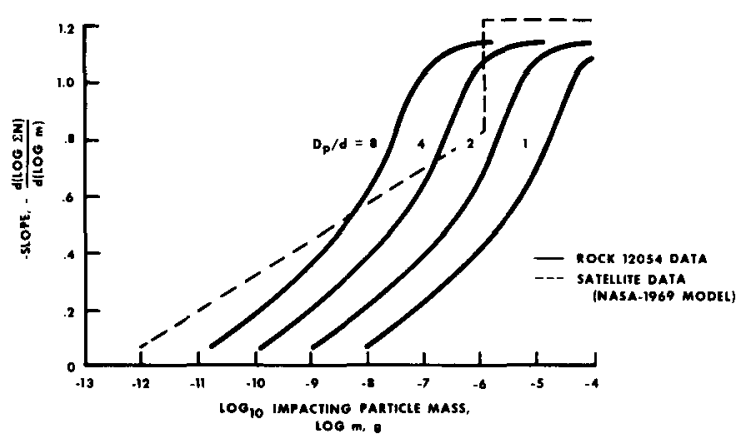

(c) SLOPE COMPARISON

FigURe 8.-Meteoroid crater density data derived from the study of rock 12054 for a range of possible $D_{p} / d$ ratios shown in (a) are compared with similar flux data based on satellite-borne experiments, shown in (b). A direct comparison cannot be made because the exposure time of the lunar rock surface is not yet known. Comparison of the slopes of the curves for the two sets of data is meaningful because the slopes indicate the relative numbers of particles without regard for absolute values for areas, times, or exposure angles. Such a comparison (c) shows agreement that there are, relatively, increasingly fewer particles with masses below about $10^{-6} \mathrm{~g}$. Lunar rock data suggest a somewhat higher minimum or cut-off particle size than the satellite data show. The angularity of the satellite data curve is artificial and due only to the equations selected to represent the data.

learn something about the mass distribution of micrometeoroids by comparing our data derived from rock 12054 with data obtained from arti- ficial-satellite-borne detection experiments. Figure 8 (b) is a log-log plot of cumulative particle flux versus particle mass, showing data from satelliteborne experiments where redundant detection devices were operating and also showing a curve representing an integrated summary of these and other data available in 1969 (Cour-Palais, 1969).

The first derivatives (slopes) of the curves in figures 8(a) and 8 (b) expressed as a function of $\log m$ may be compared directly and are plotted in figure 8(c). Assuming a constant $D_{p} / d$ value between 2 and 4 for particles with masses greater than $10^{-6}$ gram, the absolute value of the slope greater than one indicates a successively greater increase in the total number of particles for each incremental decrease in the value of $\log m$. A marked relative depletion of particles with masses below $10^{-6} \mathrm{~g}$ is clearly shown by the decreasing slope for these smaller particles. These results are in good agreement with those based on satellite-borne experiments, as is shown in figure $8(\mathrm{c})$. However, at even smaller particle sizes, the curves diverge beyond what might be considered experimental error. Our lunar rock data suggest a cut-off or almost total absence of particle smaller than $10^{-10}$ to $10^{-11}$ gram. This result disagrees somewhat with the NASA 1969 model (fig. 8(c)) and seriously with the result of Berg and Gerloff (1970), who show a considerable number of particles in this size range and smaller (fig. 8(b)).

This sharper decrease in the absolute value of the slope for the lunar rock data is probably in part due to the recognition problem discussed previously. However, we suggest that even after this effect is fully accounted for, the cut-off may still occur at a higher mass than that indicated by the satellite data. Our results are based on optical microscope observations. Scanning electron microscope studies are required to evaluate the population of extremely small micrometeoroids and thus to establish a more reliable value for the cut-off or minimum meteoroid size.

\section{METEOROID COMPOSITION}

The investigation of lunar microcraters may also yield information concerning the chemical composition of micrometeoroids. The impact- 
melted glass linings of pits may be analyzed for traces of the projectile material. Though extensive data are lacking, Bloch et al. (1971b) and Chao et al. (1970) noted no enhancement of iron or nickel relative to the surrounding host rocks, with the possible exception of one crater (Bloch et al., 1971b). Thus, it may be concluded that most of the craters are produced by projectiles of nonmetallic composition. This is in agreement with independent studies of "meteoritic contamination" of the total lunar regolith as well as selected glass coatings for which carbonaceous chondrite compositions of the projectiles were suggested by Ganapathy et al. (1970) and Morgan et al. (1971).

These results can be substantiated in a qualitative way by our microscopic obscrvations. If we consider only craters with smaller diameters than the average grain size of the host crystalline rock, or if we limit ourselves to craters which are confined to single feldspar crystals, we observe that most of these craters possess clear or transparent glass linings. Only a very small proportion have dark glass linings. If projectiles of metallic composition were abundant, we should see many more craters with dark glass linings.

This deficiency of metallic (opaque) particles may be explained by considering the PoyntingRobertson effect. It has been shown that the Poynting-Robertson effect is important in causing particles to be removed from the solar system and that the efficiency of the Poynting-Robertson effect is a direct function of the opacity of the orbiting particle. In other words, a clear particle should stay longer at a given distance from the Sun than a dark or opaque particle of the same size and density. Therefore, the number of small, clear particles should be relatively greater than the number of small, dark particles, which are under the influence of the Poynting-Robertson effect. We suggest that this effect may explain the relative excess of small clear-glass-type pits on lunar rocks.

\section{SUMMARY OF RESULTS AND FUTURE RESEARCH}

Just as we have learned about meteoroids by observing the interaction of these particles with the Earth, so we may expect to learn more about meteoroids by observing the effects of their interaction with the Moon. We have shown that the effects of single meteoroids are recorded on lunar rocks in the form of microcraters. Study of these microcraters has already produced estimates of the mass distribution of meteoroids and may be expected to produce velocity, composition, and flux information related to solid interplanetary particles.

Further experimental work is required to improve the calibration of the lunar rock meteoroid detector. Additional study of especially selected and carefully prepared lunar rock surfaces is planned to extend in either direction the range of meteoroid masses detected with confidence. Chemical analysis of pit glass from a lunar rock has been reported by Chao et al. (1970), but a much more extensive effort will be required to determine what can and cannot be learned about meteoroid composition using this approach. Finally, a successful measurement of the time of exposure of a lunar rock surface or the time since the formation of a single microcrater is of prime importance. The demonstration and application of such capabilities will lead to an independent measure of the flux of meteoroids averaged over about $10^{6}$ years. 


\section{REFERENCES}

Alexander, W. M., Corbin, J. D., Arthur, C. W., and Bohn, J. L., 1970. Picogram dust particle flux: 1967-1968 measurements in selenocentric, cislunar and interplanetary space, Space Research $X, 252-259$.

Anon., 1969. Preliminary examination of lunar samples from Apollo 11, Science, Lunar Sample Preliminary Examination Team (LSPET), 165, 1211-1227.

Berg, O. E., ANd Gerloff, U., 1970. Orbital elements of micrometeorites derived from Pioneer 8 measurements, J. Geophys. Res., 75, 6932-6939.

Bloch, R., Fechtig, H., Gentner, W., Neukum, G., Schneider, E., and Wirth, H., 1971a. Natural and simulated impact phenomena-a photo-documentation, Max-Planck-Institut für Kernphysik, Heidelberg, MPI H-1971 VI.

Bloch, R., Fechtig, H., Gentner, W., Neukum, G., and Schneider, E., 1971b. Meteorite impact craters, crater simulations and the meteoroid flux in the early solar system, Proc. Second Lunar Sci. Conf., Geochim. Cosmochim. Acta, Suppl., 2, 2639-2652.

Bogard, D. D., Funkhouser, J. G., Schaeffer, O. A., AND Zahringer, J., 1971. Noble gas abundances in lunar materials-cosmic ray spallation products and radiation ages from the Sea of Tranquility and the Ocean of Storms, J. Geophys. Res., 76, 2757-2779.

Carter, J. L., AND McKaY, D. S., 1971. Influence of target temperature or crater morphology and implications on the origin of craters on lunar glass spheres, Proc. Second Lunar Sci. Conf., Geochim. Cosmochim. Acta, Suppl., 2, 2653-2670.

Chao, E. C. T., Borman, J. A., Minkin, J. A., James, O. B., and Desborough, G. A., 1970. Lunar glasses of impact origin: physical and chemical characteristics and geologic implications, J. Geophys. Res., 75, 7445-7479.

Cour-Palais, B. G., 1969. Meteoroid Environment Model-1969 (near Earth to lunar surface), NASA SP-8013.

Crozaz, G., HaAck, U., Hair, M., Maurette, M., Walker, R., and Woolum, D., 1970. Nuclear track studies of ancient solar radiations and dynamic lunar surface processes, in Proc. A pollo 11 Lunar Science Conf., edited by A. A. Levinson, 3, 2051-2080.

Fleischer, R. L., Haines, E. L., Hart, H. R., Jr., Woods, R. T., and Comstock, G. M., 1970. The particle track record of the Sea of Tranquility, Proc. Apollo 11 Lunar Sci. Conf., Geochim. Cosmochim. Acta, Suppl., 1, 2103-2120.

Ganapathy, R., Keays, R. R., Laul, J. C., and Anders, E., 1970. Trace elements in Apollo 11 lunar rocks: implications for meteorite influx and origin of the Moon, Proc. Apollo 11 Lunar Science Conf., edited by A. A. Levinson, 2, 1117-1142.

GaUlt, D. E., 1970. Saturation and equilibrium conditions for impact cratering on the lunar surface: criteria and implications, Radio Sci., 5, 273-291.

Gürtler, C. A., ANd Grew, G. W., 1968. Meteoroid hazard near the Moon, Science, 161, 462.

Hartmann, W. K., 1970. Preliminary note on lunar cratering rates and absolute time scales, Icarus, 12, 131-133.

Hörz, F., Hartung, J. B., AND Gault, D. E., 1971a. Micrometeorite craters and related features on lunar rock surfaces, Earth and Planet. Sci. Lett., 10, 381-386.

- - Hartung, J. B., AND Gault, D. E., 1971b. Micrometeorite craters on lunar rock surfaces, J. Geophys. Res., 76, 5770-5798.

Lat, D., Macdougall, D., Wilkening, L., and Arrhenius, G., 1970. Mixing of the lunar regolith and cosmic ray spectra: evidence from particle-track studies, Proc. Apollo 11 Lunar Science Conf., edited by A. A. Levinson, 3, 2295-2304.

Mandeville, J. -C., And Vedder, J. F., 1971. Microcraters found in glass by low density projectiles, Earth and Planet. Sci. Lett., 11, 297-306.

Moore, H. J., GaUlt, D. E., ANd Heitowit, E. D., 1964. Change of effective target strength with increasing size of hypervelocity impact craters, presented to the 7th Hypervelocity Impact Symposium, Tampa, Florida.

Morgan, J. W., Laul, J. C., Ganapathy, R., and Anders, E., 1971. Glazed lunar rocks: origin by impact, Science, 172, 556-558.

Naumann, R. J., 1966. The near Earth meteoroid environment, NASA TN D-3717.

—, JEX, D. W., AND Johnson, C. L., 1969. Calibration of Pegasus and Explorer XXIII detector panels, NASA TR R-321.

Nevkum, G., Mehl, A., Fechtia, H., and Zähringer, J., 1970. Impact phenomena of micrometeorites on lunar surface materials, Earth and Planet. Sci. Lett., 8, 31-35. 
Price, P. B., and O'Sullivan, D., 1970. Lunar erosion rate and solar flare paleontology, Proc. Apollo 11 Lunar Science Conf., edited by A. A. Levinson, 3, 2351-2360.

Shommaker, E. M., Hait, M. H., Swann, G. A., Schleicher, D. L., Schaber, G. G., Sutton, R. L., Dahlem, D. H., Goddard, E. N., and Waters, A. C., 1970. Origin of the lunar regolith at Tranquility Base, Proc. A pollo 11 Lunar Sci. Conf., Geochim. Cosmochim. Acta, Suppl., 1, 2399-2412.

Shedlovsky, J. P., Honda, M., Reedy, R. C., Evans, J. C., Jr., Lal, D., Lindstrom, R. M., Delany, A. C., Arnold, J. R., Loosli, H. H., Fruchter, J. S., and Finkel, R. C., 1970. Pattern of bombardment-produced radionuclides in rock 10017 and in lunar soil, Proc. A pollo 11 Lunar Sci. Conf., Geochim. Cosmochim. Acta, Suppl., 1, 1502-1532.

Soderblom, L. A., 1970. A model for small-impact erosion applied to the lunar surface, $J$. Geophys. Res., 75, 2655-2661.

Vedder, J. F., 1971. Microcraters in glass and minerals, Earth and Planet. Sci. Lett., 11, 291-296. 